\title{
Propiedades psicométricas de la versión abreviada de la Escala de Ira al Conducir (DAS) en población española: diferencias por edad, sexo e infracciones de tráfico
}

\author{
Amaro Egea-Caparrós*, Antonio Velandrino-Nicolás, Encarna Fernández-Ros e Iván Prieto-Martínez
}

Universidad de Murcia

\begin{abstract}
Resumen: Uno de los grupos de factores asociados a los accidentes de tráfico son los factores de personalidad. En concreto, la ira en la conducción resulta uno de los potencialmente más relevantes, ya que puede motivar y elicitar conductas agresivas. Para medir este factor de personalidad, Deffenbacher, Oetting \& Lynch (1994) desarrollaron la Driving Anger Scale (DAS) y una versión reducida de la misma (de tan sólo 14 ítems). Dada la amplia difusión de esta última, debido a su validez y rapidez, también ha comenzado a ser adaptada al español (Herrero-Fernández, 2011). Esta adaptación inicial no se ha realizado en población general y presenta una estructura factorial de tres dimensiones. Nuestro estudio ha llevado a cabo una ampliación de trabajos anteriores realizando una adaptación en población general, incluyendo datos sobre infracciones cometidas por los participantes y obteniendo propiedades psicométricas adecuadas de la escala, así como una nueva propuesta en cuanto a la composición del constructo «ira en la conducción». La estructura de la versión breve ha sido explicada por tres factores: «ira ante el avance impedido por otro», «ira ante infracción de otro conductor»e «ira ante ofensa directa»; nuestra propuesta añade un nuevo factor que hemos denominado «ira ante posible sanción». Su inclusión viene apoyada por razones teóricas y prácticas.

Palabras clave: Ira al conducir, Escala; Adaptación española; Propiedades psicométricas.
\end{abstract}

\section{Introducción}

Se puede hablar de los accidentes de tráfico como un problema de salud de primera magnitud, tanto en sociedades desarrolladas como en vías de desarrollo. En los países de ingresos altos, las correspondientes tasas de mortalidad han ido descendiendo en las últimas cuatro o cinco décadas (ver datos de la OMS, 2009), en términos generales. Con todo, los accidentes de tráfico siguen constituyendo una causa importante de defunciones, traumatismos y discapacidad. Entre otros factores mencionados como causa de los accidentes estarían los factores de personalidad. Desde mediados de los años 90 se ha prestado gran atención a la hostilidad o ira en la conducción, sobre todo a partir del estudio original por Jerry L. Deffenbacher y colaboradores (1994).

La ira en conducción se ha caracterizado como un rasgo personal, específico de la situación de conducción, enmarcado dentro de la teoría de la ira estado-rasgo (Deffenbacher, Oetting, Thwaites et al., 1996) y, de forma aún más general, inserto dentro de los modelos de la interacción entre la personalidad y la situación. El interés por el estudio de la ira al conducir tiene varias razones que lo justifican. En primer lugar, si el rasgo de ira en conducción refleja una dimensión subyacente de funcionamiento de la personalidad, aquellos conductores con un alto nivel de ira la experimentarán en una mayor variedad de situaciones que aquellos con un bajo

* Dirección para correspondencia [Correspondence address]: Amaro Egea-Caparrós. Facultad de Psicología. Universidad de Murcia. Campus Espinardo. 30100 Murcia (España).E-mail: damaro@um.es
Title: Psychometric properties of the brief version of the Driving Anger Scale (DAS) in Spanish language: Differences by age, gender and traffic violations.

Abstract: The personality factors are an important group of factors associated to traffic accidents. Specifically, driving anger can be one of the more remarkable variables because it can motivate and elicit aggressive behavior. To measure this personality factor Deffenbacher, Oetting \& Lynch (1994) developed the Driving Anger Scale (DAS) and its abbreviated version (with only 14 items). Because of the widely dissemination of this short version, owing to its validity and time-saving properties, it have been accomplished its adaptation into Spanish too (Herrero-Fernández, 2011). This first Spanish adaptation did not use general population and found a three-factorial structure. In the present study we have carried out an extension to general population, including data on traffic violations. Appropriate psychometric properties and a new four-factorial structure of «driving anger» have been achieved. The three factors on previous short versions of the DAS have been called «traffic obstructions», «illegal driving», and «hostile gestures», but we have find a fourth factor we have named «possibility of being fined». Its rationale is both empirically and theoretically discussed.

Key words: Driving Anger, Scale; Spanish adaptation; psychometric properties.

nivel y de una forma más frecuente e intensa. Además, la ira puede motivar y elicitar la agresión, de forma que los conductores con alto nivel de ira también pueden implicarse en más agresiones relacionadas con el tráfico. Finalmente, dado que una ira y agresividad elevadas pueden interferir con el procesamiento controlado de la información y perturbar la ejecución en la conducción, los sujetos con un alto nivel de ira pueden mostrar altos índices de conductas arriesgadas, accidentes u otras variables relacionadas con los accidentes.

La DAS y su forma abreviada son escalas sobre ira autoinformada en un entorno específico como es la conducción, a diferencia de otras escalas o inventarios de la ira en general: NPI (Novaco, 1975, 1977), NAS (Novaco, 1994), MAI (Siegel, 1986), STAXI (Spielberger, 1988) o la sub-escala ANG del propio MMPI-2 (Butcher, Graham, Williams y Ben-Porath, 1989). La elección de la versión abreviada de la DAS responde así también al interés por evaluar los niveles de ira suscitados por una serie de situaciones específicas de conducción.

En cuanto a los estudios de validez de la forma abreviada de la DAS, un estudio con 114 estudiantes conductores (57 con alto nivel de ira y 57 con bajo nivel), a los que se seleccionó empleando la forma abreviada del DAS, mostró tamaños del efecto consistentemente grandes. Los sujetos de alto nivel de ira experimentaron ésta 3.2 veces más frecuentemente que los de bajo nivel, implicándose en conductas agresivas 4.3 veces más a menudo y en comportamientos arriesgados 2.4 veces más frecuentemente que los conductores con bajo nivel de ira (Deffenbacher, Huff, Lynch, Oetting y Salvatore, 2000). Los conductores de ira alta también 
fueron más arriesgados, agresivos y potencialmente más predispuestos a los accidentes. Estos resultados fueron replicados en otros trabajos (Deffenbacher, Lynch, Oetting y Yingling, 2001; Deffenbacher, Lynch, Filetti, Dahlen y Oetting, 2003; Lajunen y Parker, 2001).

La Escala de la ira al conducir (DAS) se ha empleado para evaluar este rasgo en conductores de diferentes países, desde EE.UU. (Deffenbacher et al., 1994), Reino Unido (Lajunen, Parker y Stradling, 1998), Francia (Villieux y Delhomme, 2007), Nueva Zelanda (Sullman, 2006), Turquía (Yasak y Esiyok, 2009), Alemania (Steffgen, Recchia y Ludewig, 2008) y España (Sullman, Gras, Cunill, Planes y FontMayolas, 2007), siendo el estudio original el de EE.UU. y los demás, de adaptación de la escala a distintos ámbitos nacionales.

En cuanto a la estructura factorial de la escala, las diferencias más destacables son las siguientes. En el caso de la investigación original (Deffenbacher et al., 1994), en un estudio con 1526 sujetos (724 hombres y 802 mujeres, con moda de 18 años) se encontraron seis factores correspondientes a las situaciones generadoras de ira en los conductores: "gestos hostiles", "conducción ilegal", "presencia policial", "conducción lenta", "descortesía" y "obstrucciones al tráfico". En el mismo trabajo, Deffenbacher y colaboradores seleccionaron una serie de ítems de cada sub-escala que tuvieran una alta correlación con la escala completa. De esta forma se obtuvo la versión breve, con un coeficiente alfa de fiabilidad de .80 y una correlación de .95 con las puntuaciones de la escala completa. Sin embargo, no se probó ninguna estructura con esta escala reducida.

Ha habido tres estudios que empleaban el DAS con población española: Sullman, Gras, Cunill, Planes y FontMayolas (2007), Gómez-Fraguela y González-Iglesias(2010) y Herrero Fernández, (2011). En el primer caso (Sullman, et al., 2007), se utilizó la escala de 33 ítems con una muestra de 371 empleados públicos de universidad (47.2\% hombres y $52.8 \%$ mujeres, entre 22 y 75 años, con $\mathrm{M}=37.5)$ y se encontraron los mismos seis factores originales pero, de nuevo, con una composición ligeramente diferente del estudio original. Curiosamente, los conductores de esta muestra ofrecieron los niveles de ira más altos ante la 'conducción ilegal' por parte de otros, cuando los conductores en los estudios en EE.UU., Reino Unido y Nueva Zelanda mostraron una ira mayor ante la 'descortesía'. También exhibieron el menor nivel de ira ante la 'presencia policial', al igual que los conductores de Reino Unido y Nueva Zelanda.

En el trabajo de Gómez-Fraguela y González-Iglesias (2010), el estudio se realizó con una muestra de mujeres jóvenes $(N=140$, entre 19 y 26 años y $M=20.94)$ empleando la misma versión que en el estudio de Sullman, et al. (2007). Sin embargo, no se trataba de un estudio de adaptación sino un estudio correlacional sobre el papel de la personalidad (medida a través del NEO PI-R) y la ira (medida mediante el STAXI-2 y el DAS) en las conductas arriesgadas de este grupo de conductoras.
El trabajo de Herrero-Fernández (2011) empleó la forma abreviada de la Escala, esto es, la versión de 14 ítems que estudió Deffenbacher en su mismo trabajo sobre la escala completa (Deffenbacher et al., 1994). Herrero-Fernández (2011) aplicó la forma abreviada del DAS a una muestra de 528 personas (estudiantes universitarios y otros) del entorno laboral y personal del autor y de dos encuestadorescolaboradores, de las cuales 187 (35.4\%) eran hombres y 341 $(64.6 \%)$ mujeres (rango $=18-<71$ años; $\mathrm{M}=27.5$ y $\mathrm{SD}=$ 10.96). Su estructura es de tres factores: "Ira al Avance Impedido por Otros" (ítems 2, 5, 7, 8, 11, 12 y 14); "Ira ante Conducción Temeraria" (ítems 1, 3, 4, 6 y 13) e "Ira ante Hostilidad Directa" (ítems 9 y 10), siendo la nomenclatura usada la misma que en el estudio con conductores del Reino Unido (Lajunen et al., 1998).

En cuanto a las diferencias de género, en el trabajo de Sullman et al. (2007) las mujeres informaron de más ira en general, así como más ira provocada por "obstrucciones al tráfico", por "conducción ilegal" y por "conducción descortés". Este resultado está en la línea de otros que encontraron diferencias similares (Lajunen y Parker, 2001; Parker et al. 2002; Sullman, 2006). En cambio, Herrero-Fernández (2011), no encontró estas diferencias con la versión abreviada de la DAS.

Por lo que se refiere a las diferencias por intervalos de edad, en el trabajo de Sullman et al. (2007) hubo diferencias entre los grupos de edad considerados $(<30,30-44,45-59$ y 60 ó +) en el nivel global de ira y en ira provocada por "conducción descortés" y "presencia policial". Así, los menores de 30 años mostraban más ira global y causada por estos dos factores, aunque aquélla no disminuía linealmente con la edad, al menos en estos dos factores ("conducción descortés" y "presencia policial").

Con la versión abreviada de la DAS, Herrero-Fernández (2011) encontró diferencias entre los grupos de edad considerados $(<30,31-44 \mathrm{y}>44)$, tanto en la escala total como en los tres factores que obtiene de esa versión (ira ante el "avance impedido por otros", "conducción temeraria" y "hostilidad directa"), en los que los menores de 30 mostraron mayor nivel de ira. No se halló interacción entre la edad y el sexo.

No ha habido estudios con la versión breve de la DAS y muestra española que consideraran la variable "infracciones". Sullman et al. (2007) consideran otras variables "descriptivas", tales como la experiencia de conducción (encontrando que los conductores con más experiencia muestran más ira por "conducción descortés") o la implicación en accidentes (hallando que un modelo de regresión con la edad, sexo, kilometraje anual y velocidad media preferida más el nivel general de ira podía predecir la implicación en accidentes).

En definitiva, existe una relativa ausencia de investigaciones con la versión abreviada de la Escala DAS en nuestro país y población no universitaria, lo cual apoya la línea del trabajo que presentamos. En él hemos empleado una herramienta de medida de los niveles de ira en conducción (la 
versión abreviada de la Escala de Ira al Conducir o D.A.S.) para evaluar los niveles de ésta en una muestra de conductores lo más representativa posible de la población general de conductores de la Región de Murcia. Se pretende mejorar la representatividad de la muestra, evitando la posible aparición de sesgo, siempre que se mantengan las propiedades psicométricas adecuadas de la escala.

Nuestro interés, por tanto, es realizar un estudio de las propiedades psicométricas de la versión reducida de la Escala de la Ira al Conducir (Deffenbacher et al., 1994) en población general española mayor de edad, reconsiderando la estructura factorial propuesta hasta la actualidad. Se estudian las diferencias en sexo, edad, así como también las posibles diferencias en cuanto a una variable potencialmente relacionada con la accidentalidad como es el haber perdido puntos debido a sanción por una infracción de tráfico.

\section{Método}

\section{Participantes}

Los participantes se seleccionaron utilizando un grupo de autoescuelas ubicadas por toda la Región de Murcia incluyendo las siete comarcas en que puede dividirse dicha región. Todas las autoescuelas contactadas accedieron a participar. La selección de los participantes de la población general fue de conveniencia, incluyéndose a aquellos que quisieron participar voluntariamente y que cumpliesen los siguientes criterios de inclusión: tener nacionalidad española, tener licencia o permiso de conducir al menos 1 año, ser conductor habitual (conducir un mínimo de cuatro días a la semana), y aunque hubieran perdido puntos del permiso no haber realizado ningún curso de recuperación de puntos, parcial o total, hasta la fecha del estudio. Todos tenían su residencia en la región, excepto $12(3.68 \%)$ que la tenían en provincias limítrofes. El nivel de estudios informado se distribuye de la siguiente manera: $3(0.9 \%)$ no tenían estudios, $49(15.0 \%)$ tenían estudios primarios, 175 tenía estudios secundarios $(53.7 \%)$ y $99(30.3 \%)$ afirmaban tener estudios universitarios. Por tanto, la muestra empleada estaba formada por personas pertenecientes a amplios sectores de la población general, aunque el muestreo efectuado no puede garantizar la completa representatividad muestral.

La muestra inicial estuvo formada por 350 personas de las que hubo que eliminar $24(6.8 \%)$ por no cumplir los criterios de inclusión mencionados, dando lugar a una muestra finalmente compuesta por 326 personas. De ellas, 142 $(43.6 \%)$ fueron mujeres y $184(56.4 \%)$ hombres. El rango de edad fue de 18 a 80 años $(\mathrm{M}=34.91$; DT $=11.22)$. Todas tenían alguna modalidad de permiso de conducir, y 54 $(16.5 \%)$ habían cometido al menos una infracción de tráfico de cualquier tipo.

\section{Instrumento}

Se administró un cuestionario que contenía la versión reducida del DAS tal como fue traducida por Caballo y Salazar (2006), la cual incluye sólo 14 ítems (Tabla 1) que pretenden evocar distintas situaciones del tráfico habitual potencialmente generadoras de ira (Tabla 1). La respuesta a todos ellos se concreta en una escala tipo Likert de 5 puntos (de 1 = Nada hasta $5=$ Mucho) según la intensidad de ira que provoca en el conductor. En el cuestionario también se recogieron preguntas sociodemográficas, así como el número de infracciones cometidas en los dos últimos años.

Tabla 1. Ítems de la versión española del DAS (traducidos por Caballo y Salazar, 2006). Estadísticos descriptivos, Fiabilidad ( $\alpha$ ) y Varianza Extraída (VE).

\begin{tabular}{|c|c|c|c|c|}
\hline Ítem & M & DT & $\alpha$ & VE \\
\hline ESCALA TOTAL & & & .83 & .43 \\
\hline FACTOR I: Ira ante avance impedido por otro & & & .76 & .29 \\
\hline $\begin{array}{l}\text { 2. Un vehículo lento en una carretera de montaña no se aparta a su derecha para dejar a } \\
\text { la gente adelantar }\end{array}$ & 2.79 & 1.17 & & \\
\hline 14. Alguien está aparcando muy lentamente y entorpece el tráfico & 2.20 & 1.04 & & \\
\hline 12. Estás parado en un embotellamiento de tráfico & 2.50 & 1.18 & & \\
\hline 11. Un ciclista está yendo por el medio de la carretera enlenteciendo el tráfico & 3.23 & 1.21 & & \\
\hline 10. Estás conduciendo detrás de un enorme camión y no te deja ver para adelantar & 2.58 & 1.18 & & \\
\hline FACTOR II: Ira ante infracción otro conductor & & & .74 & .31 \\
\hline 1. Alguien está conduciendo en zig-zag & 3.52 & 1.25 & & \\
\hline 3. Alguien da marcha atrás estando delante de ti sin mirar & 3.76 & 1.08 & & \\
\hline 8. Alguien se pasa un semáforo o una señal de stop & 3.50 & 1.27 & & \\
\hline 13. Alguien acelera cuando tratas de adelantarlo & 3.76 & 1.07 & & \\
\hline 7. Un camión va dejando caer tierra o gravilla sobre el coche que vas conduciendo & 3.49 & 1.22 & & \\
\hline FACTOR III: Ira ante ofensa directa & & & .73 & .42 \\
\hline 5. Alguien te hace un gesto ofensivo por la forma en que conduces & 3.25 & 1.36 & & \\
\hline 9. Alguien te toca el claxon por tu forma de conducir & 3.08 & 1.31 & & \\
\hline FACTOR IV: Ira ante posible sanción & & & .58 & .33 \\
\hline 4. Te toma una foto un radar de velocidad & 3.33 & 1.36 & & \\
\hline 6. Un guardia de tráfico te señala que te apartes al arcén & 2.17 & 1.28 & & \\
\hline
\end{tabular}


La traducción propuesta por estos autores resulta muy similar a la reciente traducción de Herrero (2011). De hecho, sólo dos ítems evidencian ligeras diferencias: el $\mathrm{n}^{\circ} 4$ («Te toma una foto un radar de velocidad» en la versión de V. Caballo e I.C. Salazar y «Yendo a más velocidad de la permitida, te das cuenta de que habia un radar de control de velocidad» en la versión de Herrero, 2011) y el no 10 («Estás conduciendo detrás de un enorme camión y no te deja ver para adelantan» en la versión de V. Caballo e I.C. Salazar y «Estás conduciendo detrás de un largo camión que no te deja ver alrededor de éli en la versión de Herrero, 2011). En función de esto, el equipo de investigación consensuó el uso de la versión de Caballo y Salazar (2006) al considerarla adecuada y un fiel reflejo del original.

\section{Procedimiento}

Como se ha explicado anteriormente, no se consideró necesario realizar el proceso de traducción/retrotraducción, dado que la convergencia de la versión original con la realizada por Caballo y Salazar (2006) era muy alta.

La administración del cuestionario fue llevada a cabo por los miembros de equipo investigador y fue individual o colectiva, según la disponibilidad de participantes en cada momento, así como de forma anónima y en cada uno de los locales de las autoescuelas participantes en el estudio. Las fechas en las que se administró fueron en los meses de octubre a diciembre de 2010 .

\section{Resultados}

Respecto a la composición de la muestra, se comprobó que tenía significativamente un mayor número de hombres que de mujeres, $\chi^{2}(1)=5.41, \mathrm{p}<.05$, que el número de personas disminuía significativamente conforme aumentaba el grupo de edad ( $<30$ años, de 31 a 44, y 45 y más), $\chi^{2}(2)=27.83, p$ $<.001$, y que también existía una diferente composición de los grupos en cuanto al número de personas que habían cometido infracciones frente a quienes no lo habían hecho, $\chi^{2}(1)=145.78, p<.001$. A continuación se obtuvieron los estadísticos descriptivos de cada ítem (Tabla 1) para verificar si provocaban la reacción de ira a la que aludían, ya que en caso de no hacerlo, debería ser eliminado utilizando el criterio ampliamente aceptado de Parker, Lajunen \& Stradling (1998), según el cual para que el ítem permanezca debe tener una puntuación media de al menos 1.5. Se aprecia en la Tabla 1 que todos los ítems superan dicho valor: desde el ítem 6 , que presenta una media de 2.17 (DT $=1.28$ ) hasta los ítems 3 y 13, ambos con media de 3.76 (DT $=1.08$ y DT $=$ 1.09 , respectivamente).

Seguidamente realizamos un análisis de componentes principales mediante rotación oblicua con el método Oblimin directo (con $\Delta=0$ ). Se decidió la utilización de este procedimiento debido a que se esperaba que los factores resultantes de la extracción estuvieran relacionados (Ferrando y Anguiano-Carrasco, 2010). Esta suposición se verificó, tal como se aprecia en los valores de asociación entre los facto- res obtenidos de la Tabla 2. Los criterios de aplicación del análisis factorial resultaron satisfactorios. En efecto, tanto el índice de adecuación muestral KMO que arrojó un valor de .85 , como el índice de esfericidad, $\chi^{2}(91)=1195.63, \mathrm{p}<$ .001 , indican un uso adecuado del AFE para nuestros datos. El resultado de este análisis arrojó cuatro factores principales, tal como puede verse en la Tabla 1, que explican el $59.2 \%$ de la varianza observada. En cuanto a la fiabilidad y la VE, tanto la escala total como las distintas subescalas presentan valores aceptables. Por su parte, los pesos factoriales de los ítems se presentan en la Tabla 3.

Tabla 2. Correlaciones (coeficiente r) entre los Factores y la Escala total. ( ${ }^{*} \mathrm{p}$ $<.01)$

\begin{tabular}{lllll}
$<.01)$ & I & II & III & IV \\
\hline Factores & & & & \\
\hline I & $.45^{*}$ & & & \\
II & $.44^{*}$ & $.34^{*}$ & & \\
III & $.39^{*}$ & $.17^{*}$ & $.33^{*}$ & \\
Escala total & $.84^{*}$ & $.76^{*}$ & $.67^{*}$ & $.56^{*}$ \\
\hline
\end{tabular}

Tabla 3. Pesos factoriales obtenidos mediante análisis de componentes principales con rotación Oblimin

\begin{tabular}{lllll}
\hline \multicolumn{5}{l}{ Componente } \\
\cline { 2 - 4 } Item & 1 & 2 & 3 & 4 \\
\hline 10 & .795 & & & \\
14 & .782 & & & \\
12 & .731 & & & \\
2 & .654 & & & \\
11 & .571 & & & \\
13 & & .713 & & \\
7 & & .705 & & \\
3 & & .703 & & \\
1 & & .692 & & \\
8 & & .646 & & \\
5 & & & .876 & \\
9 & & & & \\
4 & & & & \\
6 & & & & \\
\hline
\end{tabular}

A continuación, con el programa AMOS 16 realizamos un análisis factorial confirmatorio, AFC, mediante el método de máxima verosimilitud, para probar distintas composiciones factoriales del cuestionario. En concreto, sometimos a prueba el modelo de un único factor, con el que estudiar la unidimensionalidad, tal como propusieron Deffenbancher y cols. (1994); el modelo de tres factores, tal como ha sido obtenido en diversos estudios (Herrero, 2011); y, por último, un modelo de cuatro factores tal como han apuntado nuestros resultados exploratorios previos y que aparece representado en la Figura 1.

Los resultados de la bondad de ajuste para los tres modelos sometidos a análisis se presentan en la Tabla 4. Para el estudio de la adecuación del modelo de medida propuesto utilizamos una combinación de índices de bondad de ajuste (Byrne, 2010). Siguiendo la clasificación de Lévy, Martín y Román (2006) consideramos dos grupos de tales índices. Por un lado, el grupo de índices de ajuste absoluto, los cuales indican globalmente el grado de exactitud con que el modelo 
predice la matriz de covarianzas o correlaciones observada. Dentro de este grupo, el contraste Chi-cuadrado pone a prueba la hipótesis de que el modelo es no significativo. Esta prueba presenta serias dificultades debido a la falta de fiabilidad que puede mostrar en función del tamaño muestral, por lo que se aconseja acompañarlo de otros índices que contrarresten esta limitación; por tanto, el valor de probabilidad asociado, $p$, debe ser no significativo. El GFI (Goodness of Fit Index) es una transformación del estadístico Chicuadrado (Jöreskog \& Sörbom, 1990) que indica un ajuste adecuado a partir de valores superiores a 0.90. El RMSEA (Root Mean Square Error of Approximation), uno de los más recomendados, representa el ajuste esperado si el modelo fuese estimado con la población y no solo con la muestra extraída. Según Browne \& Cudeck (1993), un valor menor de 0.05 indica un buen ajuste, y valores en torno a 0.08 representan un error tolerable de aproximación a la población; por encima de 0.1 sugieren una mala aproximación. Por otro lado, el grupo de índices de ajuste incremental que comparan el modelo hipotetizado con un modelo de referencia o modelo base, que normalmente es el modelo nulo. El NFI (Normed Fit Index) evalúa la reducción proporcional en la función de ajuste entre el modelo nulo y el modelo hipotetizado. Tal ajuste se considera adecuado a partir de 0.90. El CFI (Comparative Fit Index) indica la no centralidad del modelo, y se aceptan como adecuados valores superiores a 0.90 (Hoyle, 1995). Por último, el NCS (Normed Chi-Squared) es la razón entre el valor de Chi-cuadrado y los grados de libertad del modelo. Sólo fiable para muestras grandes (como en nuestro caso), indica buen ajuste para valores inferiores a 5 . Como se aprecia en la Tabla 4, el modelo de 1 factor no cumple ningún criterio de ajuste, lo que parece indicar que como apuntaba el análisis factorial exploratorio previo- la ira informada en la conducción es un constructo formado por más de una dimensión o componente. De los otros dos modelos sometidos a prueba, el que ofrece mejores índices de ajuste es el modelo de cuatro factores, aunque el modelo de tres factores presenta algunos índices muy próximos a los valores de ajuste. Además, en el modelo de cuatro factores todos los coeficientes de regresión entre las variables latentes y sus indicadores resultaron significativos (al menos, con $\mathrm{p}<$ .05). Es decir, los indicadores incluidos para medir tales variables latentes resultan adecuados como variables observadas representativas. Todo ello nos lleva a pensar que la introducción del cuarto factor mejora la explicación de la composición de nuestro constructo.

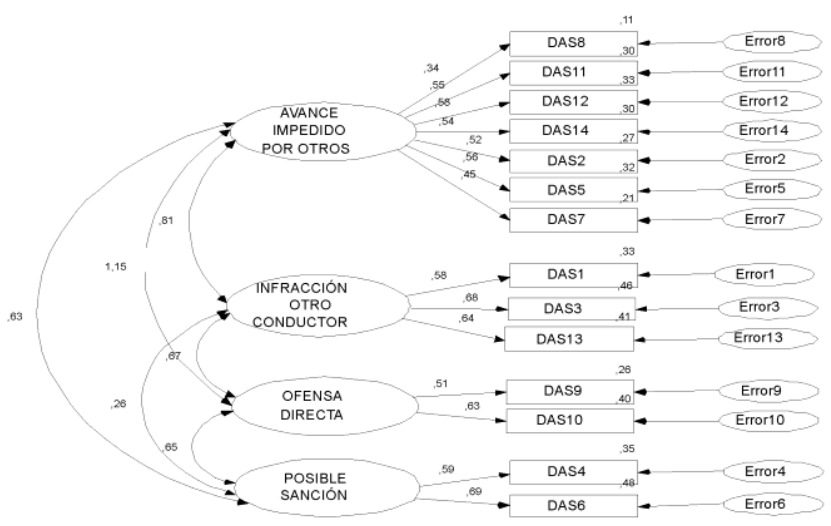

Figura 1. Path diagram del modelo de cuatro factores.

Tabla 4. Índices de Bondad de Ajuste para los tres modelos probados.

\begin{tabular}{lllllll}
\hline Modelo & $\mathrm{p}$ & $\mathrm{GFI}$ & $\mathrm{RMSEA}$ & $\mathrm{NFI}$ & $\mathrm{CFI}$ & $\chi^{2} / \mathrm{gl}$ \\
\hline Unifactorial & $<.01$ & .82 & .102 & .76 & .71 & 7.1 \\
Tres factores & $<.01$ & .84 & .089 & .88 & .85 & 4.86 \\
Cuatro factores & $<.01$ & .90 & .087 & .91 & .87 & 4.03 \\
\hline
\end{tabular}

Por último, se estudiaron las diferencias por sexo, edad y número de infracciones en cuanto al grado de ira suscitado tanto globalmente como por cada una de los cuatro factores encontrados. Como se aprecia en la Tabla 5 existen diferencias significativas en cuanto al sexo en todos los factores excepto en el factor IV de "Ira ante Posible Sanción" en el que la ira experimentada es igual entre ambos sexos. Vemos que este factor se aparta del patrón de resultados del resto de factores en el sentido de que las medias de ira no son superiores en las mujeres, sino que prácticamente se igualan. Además, como también se aprecia en dicha tabla los Tamaños del Efecto (TE) son muy bajos o casi nulos.

Tabla 5. Comparación según Sexo en la escala DAS.

\begin{tabular}{|c|c|c|c|c|c|c|c|}
\hline \multirow[t]{2}{*}{ Escala } & \multicolumn{2}{|c|}{$\begin{array}{c}\text { Hombres } \\
\mathrm{n}=184\end{array}$} & \multicolumn{2}{|c|}{$\begin{array}{l}\text { Mujeres } \\
\mathrm{n}=142\end{array}$} & \multirow{2}{*}{$\begin{array}{c}F(\mathrm{gl}) \\
(\mathrm{gl}=1 ; 324)\end{array}$} & \multirow[t]{2}{*}{$p$} & \multirow[t]{2}{*}{$\mathrm{TE}$} \\
\hline & $M$ & $D T$ & $M$ & $D T$ & & & \\
\hline Total & 41.80 & 9.04 & 44.88 & 9.64 & 8.76 & .003 & .030 \\
\hline Subescala Ira ante avance impedido por otros & 12.62 & 3.91 & 14.16 & 4.27 & 11.49 & .001 & .030 \\
\hline Subescala Ira ante infracción otro conductor & 17.62 & 4.13 & 18.56 & 4.04 & 4.27 & .040 & .011 \\
\hline Subescala Ira ante ofensa directa & 5.93 & 2.29 & 6.83 & 2.37 & 11.88 & .001 & .042 \\
\hline Subescala Ira ante posible sanción & 5.63 & 2.16 & 5.32 & 2.28 & 1.53 & .217 & .005 \\
\hline
\end{tabular}

Respecto a la comparación por edad (véase Tabla 6), al valorar la diferencia entre los tres grupos de edad (menores de 31 años, de 31 a 44 a. y mayores de 44 a.) no se encontraron diferencias estadísticamente significativas ni en la escala total ni en ninguno de los factores. Aunque no existen diferencias, se aprecia una ligera tendencia a que la ira disminuye en función del aumento de la edad. La única excepción a esta tendencia es el factor II de "Ira ante Infracción de Otro Conductor" donde la tendencia se invierte muy ligeramente, apareciendo más ira en los dos grupos de mayor edad. No obstante, los TE son prácticamente nulos. 
Tabla 6. Comparación según Edad en la escala DAS

\begin{tabular}{|c|c|c|c|c|c|c|c|c|c|}
\hline \multirow[t]{2}{*}{ Escala } & \multicolumn{2}{|c|}{$\begin{array}{c}<30 \\
\mathrm{n}=144\end{array}$} & \multicolumn{2}{|c|}{$\begin{array}{l}31-44 \\
n=115\end{array}$} & \multicolumn{2}{|c|}{$\begin{array}{c}>44 \\
n=67\end{array}$} & \multirow[t]{2}{*}{$\begin{array}{l}F \\
(\mathrm{gl}=2 ; 323)\end{array}$} & \multirow[t]{2}{*}{$p$} & \multirow[t]{2}{*}{$\mathrm{TE}$} \\
\hline & $M$ & $D T$ & $M$ & $D T$ & $M$ & $D T$ & & & \\
\hline Total & 43.57 & 9.36 & 43.23 & 8.71 & 42.07 & 10.69 & 0.58 & .569 & .004 \\
\hline Subescala Ira ante avance impedido por otros & 13.65 & 4.29 & 13.34 & 3.79 & 12.43 & 4.32 & 2.01 & .135 & .012 \\
\hline Subescala Ira ante infracción otro conductor & 17.65 & 4.0 & 18.32 & 3.91 & 18.36 & 4.63 & 1.13 & .323 & .007 \\
\hline Subescala Ira ante ofensa directa & 6.61 & 2.15 & 6.19 & 2.46 & 5.94 & 2.59 & 2.13 & .120 & .013 \\
\hline Subescala Ira ante posible sanción & 5.66 & 2.31 & 5.38 & 2.08 & 5.34 & 2.27 & 0.70 & .497 & .004 \\
\hline
\end{tabular}

En cuanto a la comparación por número de infracciones (que se dicotomizó en dos grupos: ninguna infracción y una o más infracciones), no se encontraron diferencias estadísticamente significativas (véase Tabla 7), ni en la escala global, ni en ninguno de los tres primeros factores. Solamente aparecieron diferencias en el factor IV de "Ira ante Posible Sanción”. Como se aprecia en la Tabla 7, el patrón de resultados es que la ira en este factor es mayor en las personas que cometen infracciones. Solamente en el factor II de "Ira ante Infracción de Otro Conductor" cambian ligeramente los niveles de ira, siendo ésta mayor en los no infractores. Como en las dos variables anteriores, los tamaños del efecto son muy bajos o casi nulos.

Tabla 7. Comparación según Número de Infracciones en la escala DAS

\begin{tabular}{|c|c|c|c|c|c|c|c|}
\hline \multirow[t]{2}{*}{ Escala } & \multicolumn{2}{|c|}{$\begin{array}{c}\text { Ninguna infracción } \\
\mathrm{n}=272\end{array}$} & \multicolumn{2}{|c|}{$\begin{array}{c}1 \text { o más infracciones } \\
\mathrm{n}=54\end{array}$} & \multirow{2}{*}{$\begin{array}{c}F(\mathrm{gl}) \\
(\mathrm{gl}=1 ; 324)\end{array}$} & \multirow{2}{*}{$p$} & \multirow[t]{2}{*}{$\mathrm{TE}$} \\
\hline & $M$ & $D T$ & $M$ & $D T$ & & & \\
\hline Total & 43.05 & 9.60 & 43.63 & 8.48 & 0.172 & .679 & .001 \\
\hline Subescala Ira ante avance impedido por otros & 13.24 & 4.23 & 13.56 & 3.64 & 0.263 & .608 & .001 \\
\hline Subescala Ira ante infracción otro conductor & 18.17 & 4.12 & 17.33 & 4.05 & 1.868 & .173 & .006 \\
\hline Subescala Ira ante ofensa directa & 6.27 & 2.42 & 6.59 & 2.09 & 0.826 & .364 & .003 \\
\hline Subescala Ira ante posible sanción & 5.37 & 2.26 & 6.15 & 1.89 & 5.66 & .018 & .017 \\
\hline
\end{tabular}

\section{Discusión}

Las propiedades psicométricas obtenidas en nuestra muestra apoyan la adecuación de la traducción de la versión reducida de la escala DAS como instrumento para la medición del constructo "Ira al conducir". La consistencia interna alcanza valores satisfactorios, tanto en la escala global, como en sus cuatro componentes. Los ítems presentan valores adecuados como indicadores de ira. Y la validez del constructo viene apoyada en evidencias empíricas comprobadas por los resultados de los análisis factoriales realizados. En ellos se alcanzan valores que se consideran adecuados, tanto en el AFE como en el AFC.

Se ha encontrado un nivel general de ira, medido a través de la versión abreviada del DAS, mayor en mujeres que en hombres, resultado que concuerda con el de la investigación original (Deffenbacher et al., 1994) con muestra norteamericana y la versión extensa del DAS, así como con los de Lajunen \& Parker (2001), Parker et al. (2002), Sullman (2006) y Sullman et al. (2007), si bien esas diferencias son bajas. La investigación original (Deffenbacher et al., 1994), con muestra americana y la versión completa de la DAS, mostraba que las mujeres experimentaban una ira algo mayor que los hombres sobre aspectos que interferían en su conducción, por ejemplo, conductas ilegales por parte de otros conductores, las cuales se englobarían en el Factor II encontrado en nuestro trabajo ("Ira ante infracción de otro conductor") y obstrucciones al tráfico (Factor I o "Ira ante el avance impedido por otros"). En nuestro estudio también encontramos diferencias significativas en el Factor III, de forma que las mujeres de nuestra muestra experimentan mayor ira que los hombres ante las "ofensas directas". Sin embargo, algo también interesante es que no se han encontrado diferencias en nuestro Factor IV ("Ira ante posible sanción"), contrariamente a la investigación original, en la que los hombres mostraban una ira mayor que las mujeres ante la presencia de la policía en la carretera.

Respecto al nivel de edad, no aparecen diferencias significativas en ningún factor, aunque los menores de 30 años muestran niveles algo mayores de ira. Sin embargo, la tendencia a la disminución de la ira con la edad no se mantiene en el Factor II ("Ira ante infracción de otro conductor"), en el sentido de que los conductores de 31 a 44 años y 45 o más experimentan algo más de ira que los jóvenes (menos de 30 años), aunque las diferencias no son significativas.

En cuanto a las diferencias según el número de infracciones cometidas, las comparaciones se realizaron sobre la dicotomización de esta variable entre quienes no habían cometido ninguna infracción y quienes habían cometido una o más infracciones. Dicha variable sólo mostró diferencias significativas en el Factor IV ("Ira ante posible sanción"), de manera que aquellos conductores que habían perdido puntos por haber cometido una o más infracciones experimentaban más ira en situaciones que implicaban una posible sanción, lo cual no es sorprendente. 


\section{Conclusiones}

Con los resultados obtenidos en el presente trabajo puede concluirse que la validez del constructo "Ira al conducir" queda psicométricamente apoyada en su adaptación española. Se aporta una ampliación para la explicación de dicho constructo al incluir un cuarto factor (que hemos denominado "Ira ante posible sanción") que supone una novedad respecto a estudios anteriores. Este factor parece reflejar la ira suscitada por los mecanismos de control de las vías públicas destinados a sancionar conductas no reglamentarias. La verificación definitiva de este cuarto factor (que supondría una ampliación en la definición del constructo) exige alguna evi-

\section{Referencias}

Browne, M.W. \& Cudeck, R. (1993). Alternative Ways of Assessing Model Fit. En K.A. Bollen and J. Scott-Long (Eds.), Testing Structural Equations Models (pp. 136-162). Newbury Park: Sage.

Butcher, J. N., Graham, J. R., Williams, C. L., \& Ben-Porath, Y. S. (1989). The development and use of the MMPI-2 content scales. Minneapolis, MN: University of Minnesota Press.

Byrne, B. (2010). Structural Equation Modeling with AMOS. New York: Psychology Press.

Caballo, V. \& Salazar, I.C. (2006). Traducción al español de la Escala de Ira al Conducir (Driving Anger Scale) en V. E. Caballo (coord.), Manual para la evaluación clinica de los trastornos psicológicos: trastornos de la edad adulta e informes psicológicos. Madrid: Pirámide.

Deffenbacher, J. L., Huff, M. E., Lynch, R. S., Oetting, E. R. \& Salvatore, N. F. (2000). Characteristics and treatment of high-anger drivers. Journal of Counseling Psychology, 47(1), 5-17.

Deffenbacher, J.L., Lynch, R.S., Filetti, L.B., Dahlen, E.R., \& Oetting, E.R. (2003). Anger, aggression, risky behavior, and crash-related outcomes in three groups of drivers. Behaviour Research and Therapy, 41 (3), 333-349.

Deffenbacher, J. L., Lynch, R. S., Oetting, E. R., \& Yingling, D. A. (2001). Driving anger: Correlates and a test of state-trait theory. Personality and Individual Differences, 31(8), 1321-1331.

Deffenbacher, J. L., Oetting, E. R., \& Lynch, R. S. (1994). Development of a driving anger scale. Psychological Reports, 74(1), 83-91.

Deffenbacher, J. L., Oetting, E. R., Thwaites, G. A., Lynch, R. S., Baker, D. A., Stark, R. S., Thacker, S., \& Eiswerth-Cox, L. (1996). State-trait anger theory and the utility of the Trait Anger Scale. Journal of Counseling Psychology, 43, 131-148.

Ferrando, P.J. y Anguiano-Carrasco, C. (2010). El análisis factorial como técnica de investigación en Psicología. Papeles del Psicólogo, 31, 18-33.

Gómez-Fraguela, J. A. \& González-Iglesias, B. (2010). El papel de la personalidad y la ira en la explicación de las conductas de riesgo al volante en mujeres jóvenes. Anales de Psicología, 26(2), 318-324.

Herrero-Fernández, D. (2011). Adaptación psicométrica de la versión reducida del Driving Anger Scale en una muestra española. Diferencias por edad y sexo. Anales de Psicología, 27(2), 544-549.

Hoyle, R.H. (1995). Structural Equation Modelling. Thousand Oaks, CA: Sage.

Jöreskog, F.G. \& Sörbom, D. (1990). SPSS, Lisrel 7 and Prelis, User's Guide and Reference. Chicago: SPSS Inc.

Lajunen, T. (1997). Personality Factors, Driving Style, and Traffic Safety. Ph.D. Thesis. Faculty of Arts, University of Helsinki, Helsinki.

Lajunen, T., Parker, D. (2001). Are aggressive people aggressive drivers? A study of the relationship between self-reported general aggressiveness, driver anger and aggressive driving. Accident Analysis Prevention, 33, 243255. dencia añadida proveniente de una prueba con más ítems específicos, ya que solamente han sido dos los que han aportado la evidencia empírica de este nuevo cuarto factor.

En resumen, los análisis psicométricos y el estudio diferencial de potenciales variables moduladoras (Edad, Sexo y Número de Infracciones) refuerzan la conclusión de que la solución adoptada de cuatro factores es factible y supone una novedad respecto a los estudios con este instrumento (la versión abreviada de la Escala de la Ira al Conducir o DAS). Este trabajo de adaptación a población española posibilitará el estudio posterior con poblaciones específicas de interés de una variable de personalidad potencialmente relevante para la Seguridad Vial como es la ira al conducir.

Lajunen, T., Parker, D., \& Stradling, S. G. (1998). Dimensions of driver anger, aggressive and highway code violations and their mediation by safety orientation in UK drivers. Transportation Research Part F: Traffic Psychol ogy and Behaviour (2), 107-121.

Lévy, J.P., Martín, M.T. \& Román, M.V. (2006). Optimización según estructuras de covarianza. En J.P. Lévy y J. Varela (Eds.), Modelización con Estructuras de Covarianzas en Ciencias Sociales. (pp. 11-30). Coruña: Netbiblo.

Novaco, R. W. (1975). Anger control: the development and evaluation of an experimental treatment. Lexington, MA: Lexington.

Novaco, R. W. (1977). A stress inoculation approach to anger management in the training of law enforcement. American Journal of Community Psychology, 5, 327-346.

Novaco, R. W. (1994). Anger as a risk factor for violence among the mentally disordered. In J. Monahan, \& H. Steadman (Eds.), Violence and mental disorder: developments in risk assessment ( pp. 21-60). Chicago: University of Chicago Press.

OMS (2009). Informe sobre la situación mundial de la seguridad vial. www.who.int/violence_injury_prevention/road_safety_status/2009

Parker, D., Lajunen, T., \& Stradling, S.G. (1998). Attitudinal predictors of interpersonally aggressive violations on the road. Trasportation Research Part F: Traffic Psychology and Behaviour (1), 11-24.

Parker, D., Lajunen, T., \& Summala, H. (2002).Anger and aggression among drivers in three European countries. Accident Analysis \& Prevention, 34(2), 229-235.

Siegel, J. M. (1986). The multidimensional anger inventory. Journal of Personality and Social Psychology, 51, 191-200.

Spielberger, C. D. (1988). State-trait anger expression inventory professional manual. Odessa, FL: Psychological Assessment Resources.

Steffgen, G., Recchia, S. \& Ludewig, J.L. (2008). Deffenbacher driving anger scale: Psychometric properties of a German version. International Journal of Psychology, 4, 545-545.

Sullman, M. J. M. (2006). Anger amongst New Zealand drivers. Transportation Research Part F: Traffic Psychology and Behaviour, 9(3), 173-184.

Sullman, M. J. M., Gras, M. E., Cunill, M., Planes, M., \& Font-Mayolas, S. (2007). Driving anger in Spain. Personality and Individual Differences, 42(4), 701-713.

Villieux, A., \& Delhomme, P. (2007). Driving Anger Scale, French adaptation: further evidence of reliability and validity. Perceptual and Motor Skills, 104, 947-957.

Yasak, Y., \& Esiyok, B. (2009). Anger amongst Turkish drivers: Driving Anger Scale and its adapted, long and short version. Safety Science, 47, 138144.

(Articulo recibido: 17-10-2011, revisado: 28-11-2011, aceptado: 01-12-2011) 